\title{
Physical and Engineering properties of Oil Contaminated Clay Soil
}

\author{
S. Karthigeyan ${ }^{(1)}$ \\ Associate Professor \\ Department of Civil Engineering \\ CEG, Anna University \\ Chennai, India
}

\author{
A. Ramachandran ${ }^{(2)}$ \\ PG student \\ Department of Civil Engineering \\ CEG, Anna University \\ Chennai, India
}

\begin{abstract}
Soil contamination due to spillage of various oil products on the land is a serious geo-environmental issue that adversely affects the geotechnical properties of soils. Contamination of the ground due to oil influences on both the physical and engineering properties of soils. Thus, the present paper is mainly aimed to discuss the properties of clay soil contaminated with varying percentage of oil with respect to aging. It revealed that there is a decrease in liquid limit and increase in shrinkage limit with respect to increase in the oil content. The shear strength is decreased and compressibility increased with increase in percentage of oil content of the contaminated clay soil.
\end{abstract}

Keywords - Clay, Engine oil, contamination, Shear strength.

\section{INTRODUCTION}

Soil contamination due to various oil products is a serious environmental issue. Oil leakage may takes place due to natural seepage and artificial spillage through soils. Oil spills in most cases are accidental and it occurs during the transportation of oil by both on the land and sea. Automobile garages, petrol stations and oil storage sites are the common places for soils contaminated with used engine oils. The extent of oil contamination in soil is mainly depends on the chemical composition of the contaminant and the properties of soils. An excessive oil contamination in the ground may leads to vertical settlement of oil storage tanks or cracking of pipelines lay in the oil contaminated ground. When an oil product accidentally spills over the ground surface, it infiltrates through the soil pores. Oil contaminated sandy soil particles gets slipped and reduces the angle of internal friction due to the presence of oil in the pores which leads to reduction in the shear strength. Hydraulic conductivity is a function of initial viscosity and the degree of oil saturation. The permeability characteristics of oil contaminated soils are decreased with increasing in the percentage of oil content due to a reduction of pore volume [3]. The effect of oil contamination is more significant in loose sand as compared to the dense sand [7]. The maximum dry density, frictional angle and compressive strength of oil contaminated soils are decreased with increase in the oil content [2]. Clay are chemically active soil particles and their behaviour can be altered substantially by the presence of oil in the pore fluids with variable degree [6] .The literature available in this topic reveals that the shear strength and dry density gets reduced in clay soil also due to the adsorbed oil in the clay particles [5]. The coefficient of permeability of oil contaminated clay has increased significantly due to the reduction in dielectric constant of oil than that of water [4]. However, porosity and swelling pressure of oil contaminated clay has decreased with increase in both the sorption time and the crude oil content [1]. In such a situation, major tasks are needed for remediation and reclamation of oil contaminated sites. The limited literature available in this topic indicates that there is a significant change in the properties of oil contaminated clay soils. In view of the above, it is necessary to understand the effect of oil contamination on the geotechnical properties of soils for new construction or needs to verify the stability of existing structures found in this type of oil contaminated ground surface. Hence, the present paper is mainly focused on to discuss the physical and engineering properties of oil contaminated clay soil.

\section{MATERIALS AND METHODS}

Clay soil samples were collected from Velachery, Chennai in India and it was air dried, pulverized before used for the detailed laboratory investigations. Table 1 and 2 shows the basic properties of clay soil and engine oil used in the study respectively.

TABLE I INDEX PROPERTIES OF VIRGIN CLAY SOIL

\begin{tabular}{|c|c|c|}
\hline \multirow{2}{*}{ BASIC PROPERTIES } & \multirow{2}{*}{ RESULTS } \\
\hline \multirow{2}{*}{ Grain } & Clay & 65 \\
\cline { 2 - 3 } Size (\%) & Sand & 19 \\
\cline { 2 - 3 } & Silt & 16 \\
\hline \multicolumn{2}{|c|}{ Liquid limit (\%) } & 68 \\
\hline \multicolumn{2}{|c|}{ Plastic limit (\%) } & 30 \\
\hline \multicolumn{2}{|c|}{ Plasticity Index (\%) } & 38 \\
\hline Soil classification & $\mathrm{CH}$ \\
\hline Specific Gravity & 2.73 \\
\hline Shrinkage limit (\%) & 10.6 \\
\hline Free swell index (\%) & 80 \\
\hline
\end{tabular}

TABLE II BASIC PROPERTIES OF ENGINE OIL

\begin{tabular}{|c|c|}
\hline BASIC PROPERTIES & RESULTS \\
\hline Color & Dark brown \\
\hline Density & $852 \mathrm{~kg} / \mathrm{m}^{3}$ \\
\hline Viscosity & $142 \mathrm{~mm}^{2} / \mathrm{s}$ \\
\hline
\end{tabular}

Clay soil samples were prepared by adding engine oils in terms of the varying percentage in the order of $0,3,6,9,12$, and $15 \%$ to the dry weight of the soil. The engine oil was added to the soil sample and mixed thoroughly in proportion to the weight of soil. The contaminated soil samples were exposed to the natural weather condition to study the effect of aging. The contaminated clay soil samples were tested in the 
laboratory with respect to varying aging period of 7, 14 and 28 days.

\section{RESULTS AND DISCUSSION}

Series of various laboratory tests were conducted on virgin clay soil and oil contaminated clay soils with respect to aging for evaluating the physical and engineering properties of it. The effect of oil on the physical properties and engineering properties of the clay based on the results obtained from various tests are discussed in the following headings.

\section{A. Liquid limit and plastic limit:}

Figs. 1 and 2 shows test results of liquid limit and plastic limit with respect to aging of $0,7,14$ and 28 days on the oil contaminated clay soil samples. It is seen from these figures that the liquid limit and plastic limit got decreased with increase in the oil content as compared to the virgin clay. This trend is observed to be uniform with respect to all aging periods. In general, the liquid limit and plastic limit of the oil contaminated clay is increasing with increase in aging when compared to the immediate response. However, it can be seen no much variation in the case of soil samples were tested after 7 and 14 days as compared to the immediate response. But it is interesting to see that the soil samples tested for 28 days shows a considerable increase in the liquid limit and plastic limit as compared to the immediate response. It is mainly attributes to the evaporation of oil on exposure to sunlight for longer period in case of the oil contaminated clay with 28 days aging.

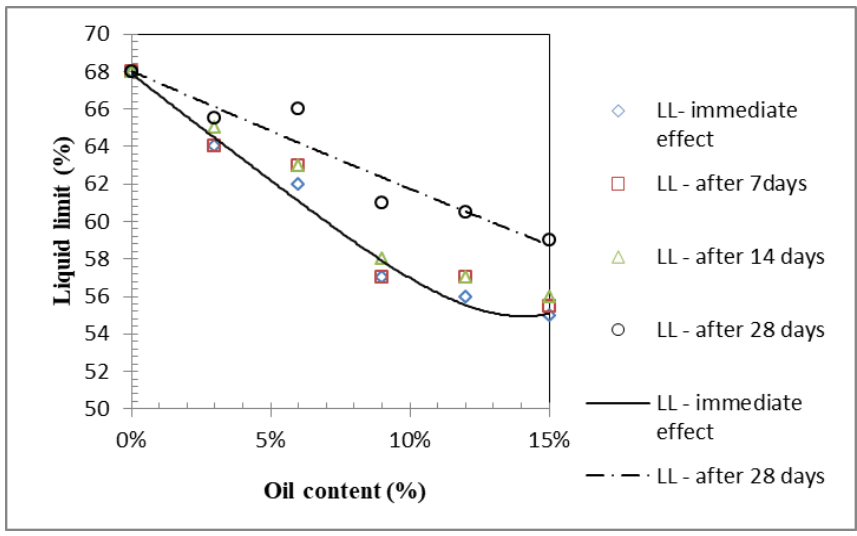

Fig. 1 Liquid limit with varying percentage of oil contamination

\section{B. Shrinkage limit and Free swell index:}

Figs. 3 and 4 shows shrinkage limit and free swell index of both the virgin and contaminated soil samples with varying percentage of oil content. It is seen from the Fig. 3 that shrinkage limit found to increase with increase in the oil content. It can be seen a similar trend for both the liquid limit and plastic limits that there is no much variation in the case of soil samples tested after 7 and 14 days of aging periods compared to the test conducted immediately after preparation the test specimen. Also, it is observed that the clay samples tested for 28 days shows a considerable decrease in the shrinkage limit as compared to the immediate response.

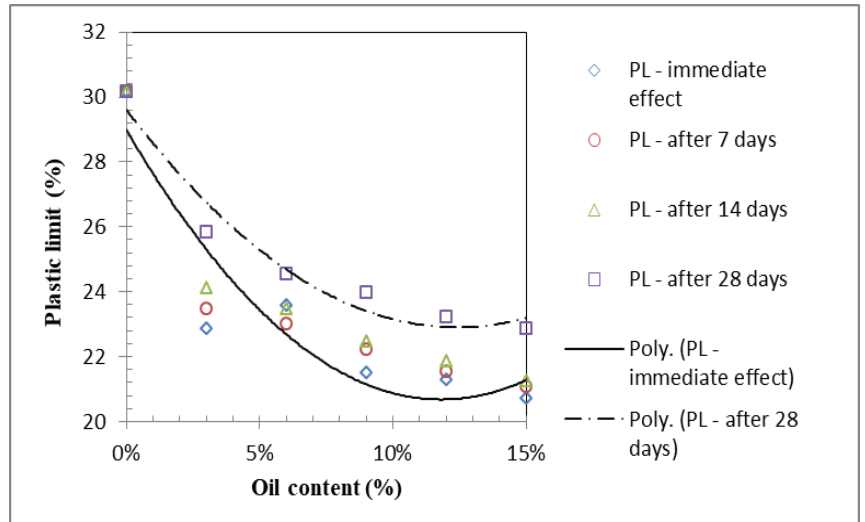

Fig. 2 Plastic limit with varying percentage of oil contamination

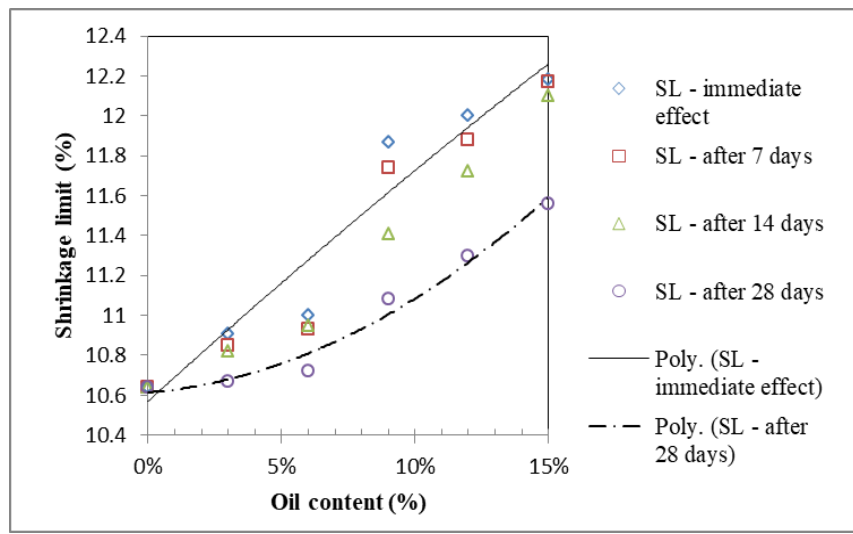

Fig. 3 Shrinkage limit with varying percentage of oil contamination

The Free Swell Index (FSI) is an indicator of the swell potential character of the soil. The FSI tests were conducted on oil contaminated clay soil samples with respect to the varying percentage of oil content and at different time periods such as immediate response after mixing and response at the end of 28 days after mixing.

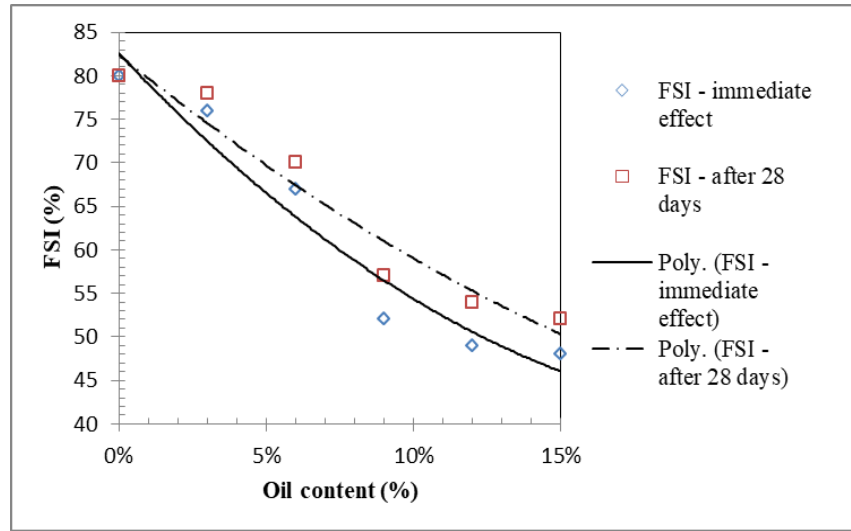

Fig. 4 FSI with varying percentage of oil contamination

Fig. 4 shows a decrease in the free swell index value with increasing in the percentage of oil content. It may be due to the reason there is a potential expansion of the double layer that gets reduced when oil surrounding the clay particles as compared to the water. It can be concluded that the swell potential of the clay is reduced by increasing the percentage of oil. However, there is an increase in the FSI for soil samples with natural exposure of drying for 28 days as compared to the immediate response of the soil. This kind of the response may 
be attributed to evaporation of the oil from the soil sample through thermal conductivity.

\section{Shear strength characteristics:}

The vane shear and unconfined compression (UCC) tests were conducted to evaluate the shear strength of both the virgin and oil contaminated clay soil samples with respect to the varying consistency and aging periods. Figs. 5 to 7 shows test results of oil contaminated clay soils with respect to three different consistencies such as stiff, medium and soft respectively. Totally 13 soil samples were tested with increase in the oil content in terms of $0,3,6,9,12$ and 15 percentage of dry soil.

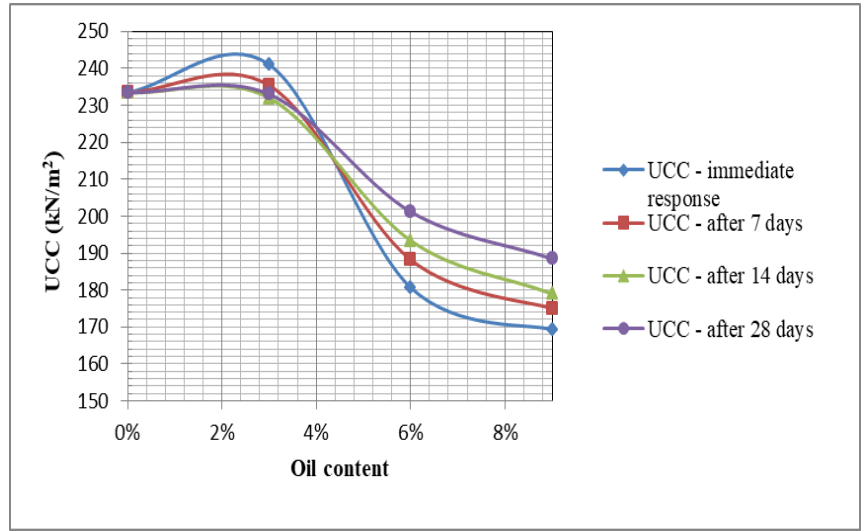

Fig. 5 Shear strength of oil contaminated clay with stiff consistency

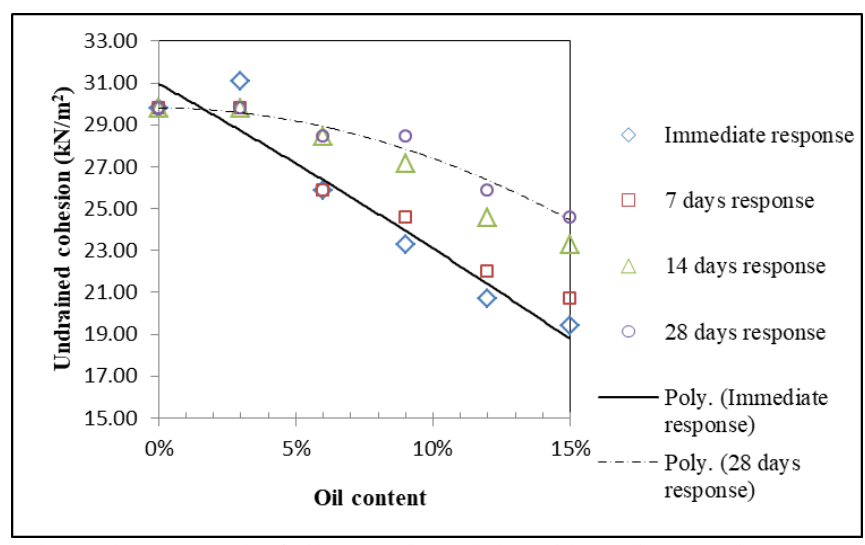

Fig. 6 Shear strength of oil contaminated clay with medium consistency

Fig. 5 shows a slight increase in the shear strength with increasing the oil content up to $3 \%$ for clay soil with stiff consistency. However, the shear strength starts decrease steeply on further addition of the oil content up to $6 \%$ and almost becomes constant at $9 \%$ of the oil content. This trend is observed to be same for the stiff clay soil samples were tested after 7, 14 and 28 days. It may be due to the reasons that all soil samples were exposed to natural drying and tested at the end of 7, 14 and 28 days. Fig. 6 shows a decrease in the shear strength with increase in the oil content up to $3 \%$ for clay with medium consistency. The shear strength of clay contaminated with $3 \%$ of the oil content is almost equal to the virgin soil and the shear strength got decreased with further increase in the oil content up to $15 \%$. The immediate response and the response at the end of 7 and 14 days shows a small increase in the shear strength but there is a considerable increase in the shear strength in case of the soil samples were tested after 28 days.

Fig. 7 shows shear strength of oil contaminated soft clay with respect to aging period. It is shown from the figure that the shear strength get decreased with increase in the oil content. The immediate response and the response at the end of 7 and 14 days shows a very small increase in the shear strength but there is a considerable increase in the shear strength in case of the soft clay samples were tested after the 28 days.

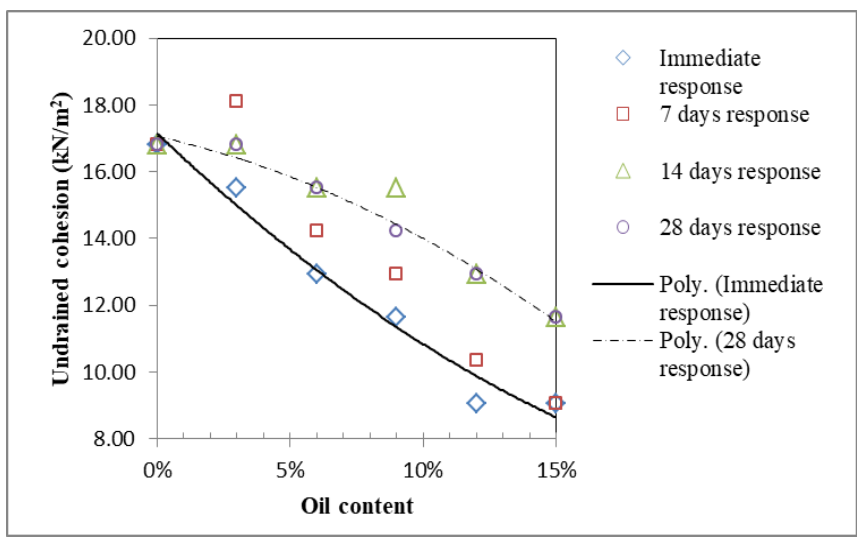

Fig. 7 Shear strength of oil contaminated clay with soft consistency

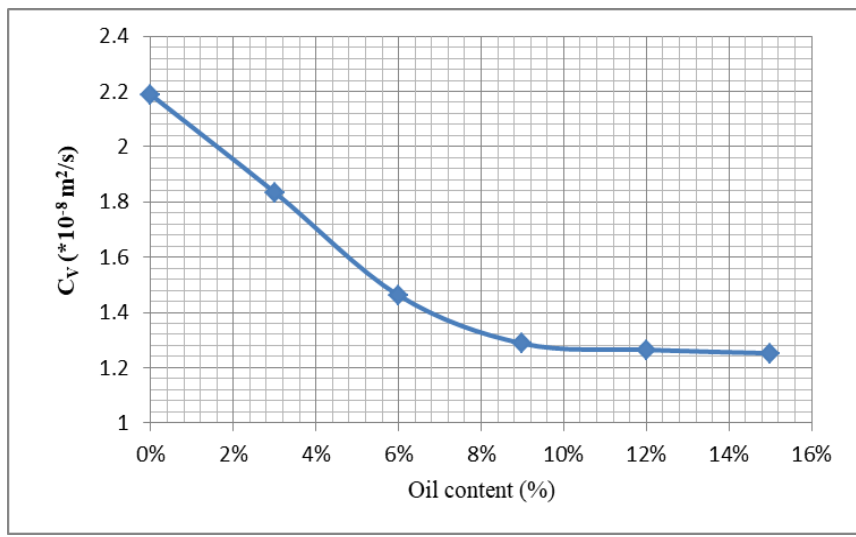

Fig. 8 Coefficient of consolidation with varying percentage of oil content

\section{Compressibility characteristics:}

A series of consolidation tests were carried out to study the compressibility characteristics of both the virgin and oil contaminated clay soil samples with soft consistency. Based on the test results, the compression index and the coefficients of consolidations were determined. Fig. 8 shows the variation of coefficient of consolidation $\left(\mathrm{c}_{\mathrm{v}}\right)$ with respect to varying percentage of the oil contamination. It is seen from the figure that the coefficient of consolidation is decreasing with increase in percentage of the oil content up to $9 \%$ and become constant with further increase in percentage of the oil content. It may be attributed that the reduction in rate of settlement in the case of oil contaminated clay as being a viscous in nature. Hence, the viscous in nature of the oil contaminated clay requires very high pressure and more time for compression as compared to the uncontaminated clay. 


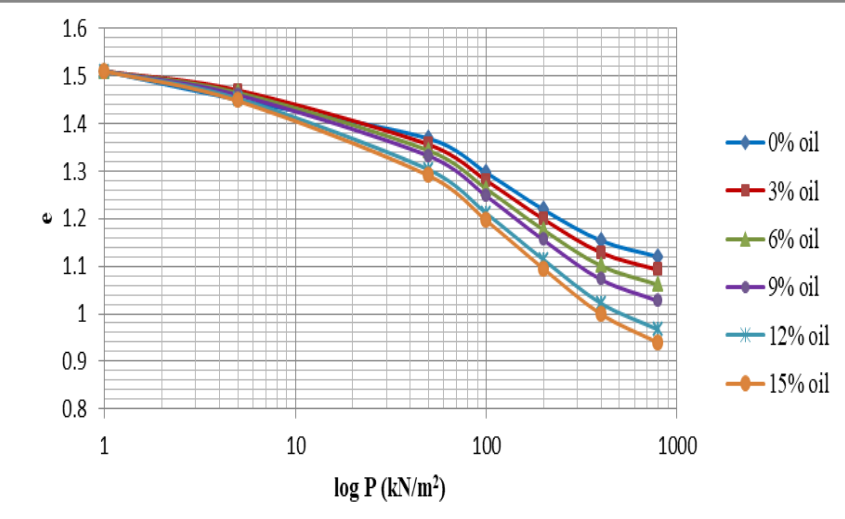

Fig. 9 Variation of void ratio with respect to applied pressure

Fig. 9 shows e - log p curve for clay contaminated with varying percentage of the oil content. It can be seen that almost a linear behavior for clay soil contaminated with the oil. This trend is observed to be same for the clay contaminated with any percentage of the oil content. Fig. 10 shows the variation of compression index for clay soil contaminated with varying percentage of the oil content. It can be seen from the figure that the compression index value is found to increase with increase in percentage of the oil content.

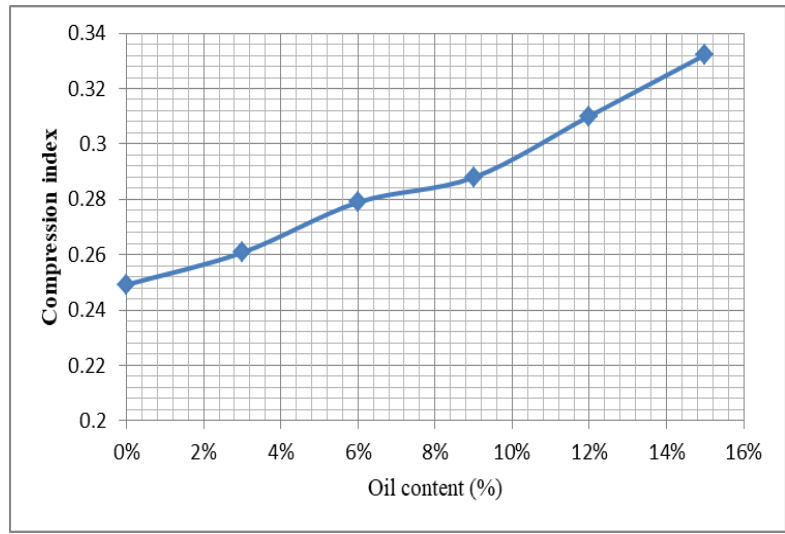

Fig. 10 Compression index with varying percentage of oil content

\section{CONCLUSION}

Based on various laboratory test results from these analyses, the following conclusions can be drawn:

\section{a) Physical properties}

[1] Liquid limit, plastic limit and free swell index values of oil contaminated clay are decreased with increase in the oil content level. However, the rate of decrease is mainly depends on the aging period.

[2] The percentage reduction in liquid limit is by a maximum of $19 \%$ in the case of immediate effect and $13 \%$ for 28 days. Similarly, the percentage reduction in plastic limit is by a maximum of $31 \%$ in the case of immediate effect and $24 \%$ for the aging period of 28 days.
[3] The percentage reduction in free swell index of the oil contaminated clay soil is by a maximum of $40 \%$ in the case of immediate effect and $35 \%$ for the aging period of 28 days.

[4] Shrinkage limit is increased with increase in the oil content level. The percentage increase in shrinkage limit of oil contaminated clay soil is by a maximum of $14 \%$ in the case of immediate effect and $9 \%$ for the aging period of 28 days.

[5] It can be concluded from the shrinkage limit and FSI, the swelling potential for oil contaminated clay is decreased with increase in the oil content level.

\section{b) Engineering properties}

[1] Shear strength of oil contaminated clay is increasing with increase in the oil content level up to $3 \%$ with further increase in oil content shown a reduction in the shear strength. This trend is common for oil contaminated clay of different consistencies.

[2] The effect of aging is significantly influences on the geotechnical properties of oil contaminated clay. The shear strength of the oil contaminated clay is decreased with increase in the aging period.

[3] The percentage reduction in the shear strength of oil contaminated clay is by a maximum of $27 \%$ to 46 $\%$ in the case of immediate effect and $17 \%$ to $30 \%$ for the aging period of 28 days.

[4] The compression index value of oil contaminated clay is increased with increase in the oil content level. The percentage increase in the compression index value is by a maximum of $33 \%$.

[5] The rate of consolidation is decreased with increase in the oil content level. The percentage reduction in coefficient of consolidation is by a maximum of 42 $\%$.

\section{REFERENCES}

[1] Adejumo T. E., "Effect of crude oil contamination on the geotechnical properties of soft clay soils ofniger delta regions of Nigeria”, Electronic Journal of Geotechnical Engineering, 2012, volume No.17, pp. 1929-1938.

[2] Al-Duwaisan D. B. and Al-Naseem A. A.,"Characterization of oil contaminatedsoil", $2^{\text {nd }}$ International conference on Environmental Science and Technology, 2011,Volume No. 6, Issue No. 2, pp. 439-442.

[3] Khamehchiyan M., Charkhabi A.H. and Tajik M., "Effect of crude oil contamination on geotechnical properties of clayey and sandy soils", Journal of Engineering Geology, 2007, Volume No. 89, pp. 220-229.

[4] Nazir A.K., "Effect of motor oil contamination on geotechnical properties of over consolidated clay", Alexandria Engineering Journal, 2011, Volume No.123, pp. 331-335.

[5] Pandey A. and Bind Y.K., "Effect of oil contamination on the geotechnical properties of alluvial soil Naini, Allahabad”, International Journal of Innovative Technology and Exploring Engineering, 2014,volume No.3,Issue no.8..

[6] Swaroop s.s. and Rani V, Effect of Oil Contamination on Geotechnical Properties of Clayey SoilInternational Journal of Engineering Research \& Technology (IJERT), ISSN: 2278-0181, NCRACE-2015 Conference Proceedings.

[7] Umanath U. and Karthigeyan S., 'Geotechnical characteristics of oil contaminated sand by oil spillage', ACIDIC-2014, National Institute of Technology Karnataka, Surathkal,, 2014,Volume No. 1, pp. 387-393. 